\title{
Coulomb Blockade Oscillations of Conductance at Finite Energy Level Spacing in a Quantum Dot
}

\author{
Serguei Vorojtsov \\ Department of Physics, Duke University, Durham, NC 27r08-0305
}

(Dated: October 30, 2018)

\begin{abstract}
We find an analytical expression for the conductance of a single electron transistor in the regime when temperature, level spacing, and charging energy of a grain are all of the same order. We consider the model of equidistant energy levels in a grain in the sequential tunneling approximation. In the case of spinless electrons our theory describes transport through a dot in the quantum Hall regime. In the case of spin- $\frac{1}{2}$ electrons we analyze the line shape of a peak, shift in the position of the peak's maximum as a function of temperature, and the values of the conductance in the odd and even valleys.
\end{abstract}

PACS numbers: 73.23.Hk, 73.23.-b, 73.43.Jn

\section{INTRODUCTION}

Recent progress in mesoscopic fabrication techniques has made possible not only the creation of more sophisticated devices but also greater control over their properties. Electron systems confined to small space regions, quantum dots, and especially their transport properties have been studied extensively for the last decade. ${ }^{1.2}$ In particular, an individual ultra-small metallic grain of radius less than $5 \mathrm{~nm}$ was attached to two leads via oxide tunnel barriers, thus forming a single electron transistor (SET) $\underline{\underline{1}}$ Applying bias voltage, $V$, between two leads allows one to study transport properties of the system, Fig. 11 Alternatively, a SET can be formed by depleting two-dimensional electron gas at the interface of GaAs/AlGaAs heterostructure by applying negative voltages to the metallic surface gates: ${ }^{2}$

In this paper we will assume that the bias voltage is infinitesimally small, $V \rightarrow 0$. This corresponds to the linear response regime. In order to tunnel onto the quantum dot, an electron in the left lead has to overcome a charging energy, $E_{C}=e^{2} / 2 C$, where $e>0$ is the elementary charge; $C$ is the capacitance of the quantum dot. If $T \ll E_{C}$ then conductance through the system is exponentially suppressed. This phenomenon is called the

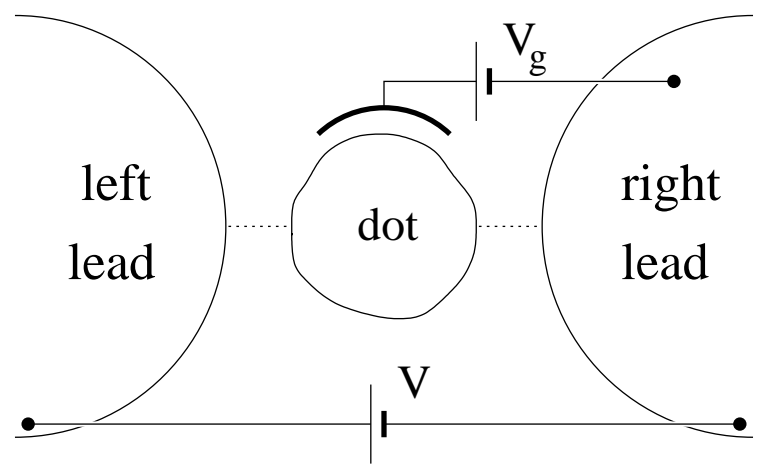

FIG. 1: Scheme of the Coulomb blockade setup.
Coulomb blockade. However if we apply a voltage, $V_{g}$, to the additional gate capacitively coupled to the dot, the Coulomb blockade can be lifted. Indeed, changing $V_{g}$ one can shift the position of energy minimum so that energies of the quantum dot with $N_{e}$ and $N_{e}+1$ electrons will become equal and an electron can freely jump from the left lead onto the dot and then jump out into the other lead. Thus, current event has occurred and a peak in the conductance, $G$, corresponding to this gate voltage is observed. By changing the gate voltage one can observe an oscillation of the conductance or Coulomb blockade oscillations.

One-particle energy levels in the quantum dot, $\left\{E_{i}\right\}$, are given by the solution of the Schrödinger equation in the quantum dot's potential. The mean spacing between these energy levels is $\delta E$. The conventional assumption that $E_{C} \gg \delta E$ is not valid in the case of sufficiently small dots. In fact, in the recent experiments,, 3.4 where a $C_{60}$ molecule has acted as a quantum dot, the level spacing is of order charging energy. Experiment ${ }^{3}$ was performed at $T=4.2 \mathrm{~K}$ as well as at room temperature. In other experiments $s^{5.6}$ with quantum dot formed by depleting $2 \mathrm{DEG}^{5}$ and ropes of carbon nanotubes acting as a quantum dot,,$\frac{6}{}$ charging energy is only three times larger than the spacing $\delta E$.

Though Coulomb blockade oscillations have been studied in a number of important limiting cases,,$\frac{7,8,10}{}$ the problem in the case when values of $E_{C}, \delta E$, and $T$ are all of the same order has not been theoretically addressed. Let us note that energy levels of the quantum dots are random and obey Wigner-Dyson statistics with the fluctuation of order of their mean $\stackrel{11}{*}$ Nonetheless to go as far as possible in the analytical treatment of the problem we have to assume that energy levels in the quantum dot are equidistant. In this paper we derive an analytical expression for the linear conductance, $G=I /\left.V\right|_{V \rightarrow 0}$, in the case of spinless as well as spin- $\frac{1}{2}$ fermions.

In Sec. II, we describe our model and the assumptions involved. We write the model assuming spin- $\frac{1}{2}$ fermions. In Sec. III, we consider the linear conductance in the case of spinless fermions. We obtain an analytical expression 
for the conductance and analyze its limiting cases. In Sec. IV, we consider one possible application of the Sec. III results, namely tunneling through the edge states in a quantum dot placed into a strong magnetic field. In Sec. V, the linear conductance as well as its properties in the case of spin- $\frac{1}{2}$ fermions is considered. In Sec. VI, we summarize our findings.

\section{THE MODEL}

Hamiltonian of the system in question is

$$
\hat{H}=\hat{H}_{l}+\hat{H}_{d}+\hat{T} .
$$

Here, the first term is the Hamiltonian of noninteracting electrons in the left and right leads:

$$
\hat{H}_{l}=\sum_{k \sigma} E_{k} c_{k \sigma}^{\dagger} c_{k \sigma}+\sum_{p \sigma} E_{p} c_{p \sigma}^{\dagger} c_{p \sigma}
$$

where a continuum of states in each lead, $|k \sigma\rangle,|p \sigma\rangle$ is assumed; $E_{k}, E_{p}$ and $c_{k \sigma}, c_{p \sigma}$ are the energies and electron annihilation operators in the left and right leads, respectively; $\sigma$ stands for the $z$-component of spin. The chemical potentials of the leads, $\mu \gg E_{C}, \delta E, T$, are shifted according to the bias voltage, $V$, applied, Fig. 2 We will assume that leads are in thermal equilibrium at temperature $T$ and, thus, occupied according to the Fermi-Dirac distribution.

The second term in (1) is the Hamiltonian of the quantum dot:

$$
\hat{H}_{d}=\sum_{i \sigma} E_{i} c_{i \sigma}^{\dagger} c_{i \sigma}+\hat{U}
$$

where first term is the kinetic energy of electrons in the quantum dot: $\left\{E_{i}\right\}$ is a discrete set of the quantum dot's energy levels; $c_{i \sigma}$ 's are the annihilation operators. The second term, $\hat{U}$ describes the electron-electron interaction in the quantum dot. We adopt the simplest model for the interaction, namely, the constant interaction model. In this model the Coulomb interaction of the

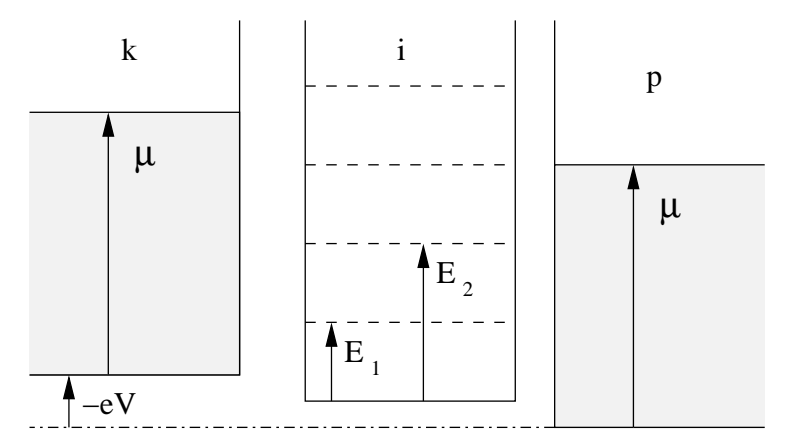

FIG. 2: Electrostatic potential energy along a line through the tunnel junctions. electrons depends only on the total number of electrons in the quantum dot:

$$
U(\hat{N})=E_{C} \hat{N}^{2}-e V_{e} \hat{N},
$$

where $N=\sum_{i \sigma} c_{i \sigma}^{\dagger} c_{i \sigma}-N_{i}$ is the total number of excess electrons; $N_{i}$ is the total number of positively charged ions. The second term is the contribution from external charges. They are supplied by the ionized donors and the gate: $V_{e}=V_{d}+a V_{g}$, where $a$ is a function of the capacitance matrix elements of the system. Thus, $V_{e}$ can be varied continuously by changing gate voltage, $V_{g}$. $U(N)$ can be rewritten as

$$
U(N)=E_{C}\left(N-N_{g}\right)^{2}+\text { Const, }
$$

where $N_{g}=e V_{e} / 2 E_{C}$ is the dimensionless gate voltage.

The third term in (1) is the tunneling Hamiltonian:

$$
\hat{T}=\sum_{k i \sigma}\left(t_{k i} c_{k \sigma}^{\dagger} c_{i \sigma}+\text { h.c. }\right)+\sum_{p i \sigma}\left(t_{p i} c_{p \sigma}^{\dagger} c_{i \sigma}+\text { h.c. }\right),
$$

where $t_{k i}$ and $t_{p i}$ are matrix elements of tunneling into the left and right leads, respectively.

We assume that the dot is weakly coupled to the leads; that is, the conductances of the dot-lead junctions are small: $G^{l, r} \ll e^{2} / h$, where $h$ is Planck's constant. Equivalently, the widths of the quantum dot's energy levels contributing to the conductance, $\Gamma_{i}=\Gamma_{i}^{l}+\Gamma_{i}^{r}$, must be small compared to spacing between them: $\Gamma_{i} \ll \delta E$. This, together with $\Gamma_{i} \ll T$ assumption, allows us to characterize the state of the dot by a set of occupation numbers, $\left\{n_{i \sigma}\right\}, 10$

\section{LINEAR CONDUCTANCE IN THE SPINLESS CASE}

The model formulated above has been studied by Beenakker in the sequential tunneling approximation, that is, conservation of energy was assumed in each tunneling process, and cotunneling was neglected. Therefore, to find the stationary current, kinetic equation considerations can be applied. In the linear response regime an analytical formula for the conductance has been obtained. In the case of spinless fermions: $\underline{\underline{10}}$

$$
\begin{aligned}
G= & \frac{e^{2}}{h T} \sum_{i=1}^{\infty} \frac{\Gamma_{i}^{l} \Gamma_{i}^{r}}{\Gamma_{i}^{l}+\Gamma_{i}^{r}} \sum_{N_{e}=1}^{\infty} P_{e q}\left(N_{e}\right) F_{e q}\left(E_{i} \mid N_{e}\right) \\
& \times\left[1-n_{F}\left(E_{i}-\mu+U(N)-U(N-1)\right]\right.
\end{aligned}
$$

where

$$
\Gamma_{i}^{l}=2 \pi \sum_{k}\left|t_{k i}\right|^{2} \delta\left[E_{i}-E_{k}+U(N)-U(N-1)\right]
$$

and

$$
\Gamma_{i}^{r}=2 \pi \sum_{p}\left|t_{p i}\right|^{2} \delta\left[E_{i}-E_{p}+U(N)-U(N-1)\right]
$$


are widths of the quantum dot's level $i$ associated with tunneling into the left and right leads, respectively; $P_{e q}\left(N_{e}\right)$ is the equilibrium probability that the quantum dot contains $N_{e}$ electrons; $F_{e q}\left(E_{i} \mid N_{e}\right)$ is the occupation number of level $i$ given that the dot contains $N_{e}$ electrons; $n_{F}(E)$ is the Fermi-Dirac distribution; and $\mu$ is the chemical potential in the leads.

The quantity $F_{e q}\left(E_{i} \mid N_{e}\right)$ in (7) is the most non-trivial one to calculate. It is the occupation number of the level $i$ in the canonical ensemble ( $N_{e}$ is fixed). In the limit $\delta E / T \rightarrow 0, F_{e q}\left(E_{i} \mid N_{e}\right)$ becomes a Fermi-Dirac distribution with the appropriately chosen chemical potential: $\tilde{\mu}=\left(E_{0}+E_{1}\right) / 2$, where $E_{0}$ corresponds to the energy of the last occupied energy level at $T=0$, Fig. [3]; $E_{1}$ corresponds to the energy of the first empty energy level at $T=0$. In the opposite limit $\delta E / T \rightarrow \infty$, the Fermi-Dirac distribution with $\tilde{\mu}=\left(E_{0}+E_{1}\right) / 2$ apparently breaks down: the occupation number of level $j=1$, for example, see Fig. [3a, is $n_{1}=e^{-\delta E / T}$, not $e^{-\delta E / 2 T}$ as the Fermi-Dirac distribution would predict 10

The occupation number in question is 10

$$
\begin{aligned}
F_{e q}\left(E_{i} \mid N_{e}\right) & =\frac{1}{P_{e q}\left(N_{e}\right)} \sum_{\left\{n_{l}\right\}} P_{e q}\left(\left\{n_{l}\right\}\right) \delta_{n_{i}, 1} \delta_{N_{e}, \sum n_{l}} \\
& =e^{\beta F\left(N_{e}\right)} \sum_{\left\{n_{l}\right\}} e^{-\beta \sum E_{l} n_{l}} \delta_{n_{i}, 1} \delta_{N_{e}, \sum n_{l}}
\end{aligned}
$$

where $P_{e q}\left(\left\{n_{l}\right\}\right)$ is the equilibrium probability of the $\left|\left\{n_{l}\right\}\right\rangle$ state of the quantum $\operatorname{dot} ; \beta=1 / T$; and the detailed definition of $F\left(N_{e}\right)$ will follow. The reason for writing this equation is to show that analytical calculation of the occupation numbers is hardly possible for arbitrary quantum dot's energy level structure, $\left\{E_{i}\right\}$.

The only way to overcome this difficulty is to assume that energy levels in the quantum dot are equidistant. Then one can use the bosonization technique ${ }^{13}$ (see Appendix) to find the exact analytical expression for the occupation numbers in the canonical ensemble. It was

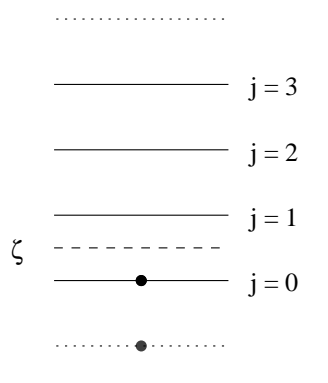

a)

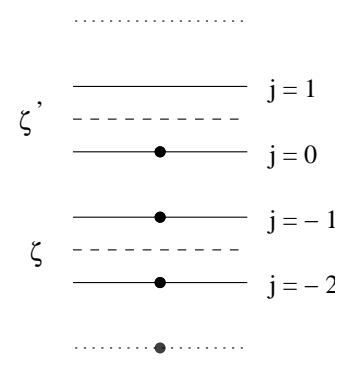

b)
FIG. 3: a) occupation numbers in the canonical ensemble for the equidistant energy levels at $T=0 ; \mathrm{b}$ ) mapping sum over $i$ onto sum over $j(N=2$ case is shown). done by Denton, Muhlschlegel, and Scalapino: ${ }^{12}$

$$
F_{e q}\left(E_{i} \mid N_{e}\right) \equiv n_{j}=\sum_{m=1}^{\infty}(-1)^{m-1} e^{-\frac{1}{2}\left[m^{2}+(2 j-1) m\right] \frac{\delta E}{T}},
$$

where

$$
j=\frac{E_{i}-\zeta}{\delta E}+\frac{1}{2}=\text { integer }
$$

$\delta E / T \equiv \delta=$ const, $\zeta$ is the energy corresponding to the highest occupied energy level at $T=0$ plus $\delta E / 2$, Fig. 3]. This quantity $\zeta$ is somewhat similar to the chemical potential of a dot, though, strictly speaking, the chemical potential is not well-defined for a dot in the canonical ensemble. The difference, $\zeta-\mu$, is a linear function of the gate voltage. Therefore, by properly adjusting "zero" value of the gate voltage it can put to zero. Hereinafter, we assume that $\zeta-\mu=0$.

To calculate the conductance we also need to know $P_{e q}\left(N_{e}\right)$, the probability that the dot, in thermodynamic equilibrium with the reservoirs, contains $N_{e}$ electrons. It can be calculated in the grand canonical ensemble:

$$
P_{e q}\left(N_{e}\right)=\frac{1}{Z_{\mu}} e^{-\beta \varphi\left(N_{e}\right)},
$$

where $Z_{\mu}=\sum_{N_{e}=0}^{\infty} e^{-\beta \varphi\left(N_{e}\right)}$ is the grand partition function; $\varphi\left(N_{e}\right)$ is the thermodynamic potential of the quantum dot. It can be expressed via free energy of the dot's internal degrees of freedom, $F\left(N_{e}\right)$ :

$$
\varphi\left(N_{e}\right)=F\left(N_{e}\right)-\mu N_{e}+U(N)
$$

hence,

$$
P_{e q}\left(N_{e}\right)=\frac{1}{Z_{\mu}} e^{-\beta U(N)} Z\left(N_{e}\right)
$$

where

$$
Z\left(N_{e}\right)=e^{-\beta\left[F\left(N_{e}\right)-\mu N_{e}\right]}=\sum_{\left\{n_{i}\right\}} e^{-\beta \sum\left(E_{i}-\mu\right) n_{i}} \delta_{N_{e}, \sum n_{i}}
$$

is the partition function in the canonical ensemble. In the last expression the sum is taken over all possible states, $\left|\left\{n_{i}\right\}\right\rangle$ of the quantum dot. To calculate $Z\left(N_{e}\right)$ explicitly we need to assume that energy levels of the dot are equidistant:

$$
Z\left(N_{e}\right)=e^{-\left(N_{e}-N_{i}\right)^{2} \delta / 2} Z_{e x c}
$$

where $N_{e}=N_{i}$ corresponds to the equilibrium number of the excess electrons $(\zeta=\mu) ; Z_{\text {exc }}$ is the partition function of the thermal excitations. Now, let us substitute Eq. (14) in Eq. (13):

$$
\begin{aligned}
P_{e q}\left(N_{e}\right) & =\frac{1}{Z_{\mu}} e^{-\beta U(N)} e^{-\left(N_{e}-N_{i}\right)^{2} \delta / 2} Z_{e x c} \\
& =\frac{1}{D_{0}^{\prime}} e^{-\beta\left[U(N)+N^{2} \delta E / 2\right]},
\end{aligned}
$$


where

$$
D_{0}^{\prime}=\sum_{N=-\infty}^{\infty} e^{-\beta\left[U(N)+N^{2} \delta E / 2\right]}
$$

Here, we have extended one limit of the sum to infinity since the Fermi energy is the largest energy scale of the problem.

Thus, we are well-equipped to calculate the conductance in the case of equidistant energy levels in the quantum dot at arbitrary ratio $\delta$. The widths of energy levels, $\Gamma_{i}^{l}$ and $\Gamma_{i}^{r}$, in the quantum dot are energy dependent, random quantities. Let us assume that quantum dot is weakly coupled to the leads via multichannel tunnel junctions: $G^{l, r}=G_{1}^{l, r} N_{c h} \ll e^{2} / h$, where $N_{c h}$ is the number of channels; $G_{1}^{l, r}$ is the conductance of one channel. Experimentally, this situation corresponds to the metallic grain coupled to the leads via oxide tunnel barriers $\stackrel{1}{-1}$ This setup allows one to decrease fluctuations of the energy levels' widths, $\Gamma_{i}^{l}$ and $\Gamma_{i}^{r}$, by a factor of $\sqrt{N_{c h}}$. We also assume that the widths are slowly changing functions of the energy, $E_{i}$. Then, $\Gamma_{i}^{l}$ and $\Gamma_{i}^{r}$ can be considered constants and evaluated at the chemical potential: $\Gamma_{i}^{l} \approx \Gamma_{\mu}^{l} \equiv \Gamma^{l} ; \Gamma_{i}^{r} \approx \Gamma_{\mu}^{r} \equiv \Gamma^{r}$. There is a simple relation between these widths and conductances of the corresponding junctions. In the case of spinless fermions:

$$
\Gamma^{l}=\frac{h G^{l}}{e^{2}} \delta E, \quad \Gamma^{r}=\frac{h G^{r}}{e^{2}} \delta E .
$$

Let us substitute Eq. (15) in Eq. (7):

$$
\begin{aligned}
G= & \frac{G^{l} G^{r}}{G^{l}+G^{r}} \frac{\delta}{D_{0}^{\prime}} \sum_{N} e^{-\beta\left[U(N)+N^{2} \delta E / 2\right]} \sum_{i} F_{e q}\left(E_{i} \mid N_{e}\right) \\
& \times\left\{1-n_{F}\left[E_{i}-\mu+U(N)-U(N-1)\right]\right\} .
\end{aligned}
$$

To take advantage of the expression for occupation numbers, Eq. (9), we need to map the sum over $i$ onto the sum over $j$. As illustrated in Fig. 3 the mapping rule depends on the total number of excess electrons, $N$ (compare with Eq. (10) written for $N=0$ ):

$$
\begin{aligned}
F_{e q}\left(E_{i} \mid N_{e}\right) & =n_{j} \\
E_{i}-\mu=E_{i}-\zeta^{\prime}+\left(\zeta^{\prime}-\zeta\right) & =\left(j-\frac{1}{2}\right) \delta E+N \delta E
\end{aligned}
$$

where $\zeta^{\prime}=\zeta^{\prime}(N)$ is the energy of the highest occupied energy level in the dot with $N$ excess electrons at $T=0$ plus $\delta E / 2$. We will also use the following identities:

$$
\begin{array}{r}
U(N)+\frac{\delta E}{2} N^{2}=\left(E_{C}+\frac{\delta E}{2}\right) N^{2}-e V_{e} N \\
=\left(E_{C}+\frac{\delta E}{2}\right)\left(N-\Delta_{0}+\frac{1}{2}\right)^{2}+\mathcal{C}_{1},
\end{array}
$$

where $\mathcal{C}_{1}=-\left(e V_{e}\right)^{2} / 2\left(2 E_{C}+\delta E\right)$;

$$
\Delta_{0} \equiv \frac{e V_{e}}{2 E_{C}+\delta E}+\frac{1}{2}
$$

has been chosen so that $\Delta_{0}=0$ corresponds to the maximum of the conductance peak; and

$$
\begin{aligned}
& U(N)-U(N-1)=(2 N-1) E_{C}-e V_{e} \\
= & (2 N-1) E_{C}-\left(2 E_{C}+\delta E\right)\left(\Delta_{0}-1 / 2\right) .
\end{aligned}
$$

Substituting these results in Eq. (18), we obtain

$$
\frac{G\left(\Delta_{0}\right)}{G_{\infty}}=\frac{\delta}{D_{0}} \sum_{N} e^{-\varepsilon_{0}\left(N-\Delta_{0}+\frac{1}{2}\right)^{2}} \sum_{j} \frac{n_{j}}{e^{-j \delta-2\left(N-\Delta_{0}\right) \varepsilon_{0}}+1},
$$

where

$$
D_{0}=\exp \left(\beta \mathcal{C}_{1}\right) D_{0}^{\prime}=\sum_{N} e^{-\varepsilon_{0}\left(N-\Delta_{0}+\frac{1}{2}\right)^{2}}
$$

$\varepsilon_{0} \equiv \beta\left(E_{C}+\delta E / 2\right) ; G_{\infty} \equiv G^{l} G^{r} /\left(G^{l}+G^{r}\right)$ is the classical, $E_{C}, \delta E \ll T \ll \mu$, limit of the conductance. We have also used the identity: $1-n_{F}(E)=\left(1+e^{-\beta E}\right)^{-1}$. Formula (22) is the general expression for the linear conductance in the spinless case for equidistant energy levels in the quantum dot at arbitrary values of $E_{C}, \delta E$ and $T$.

One can immediately prove the following properties of the conductance (22). First of all, $G\left(\Delta_{0}\right)=G\left(\Delta_{0}+M\right)$, where $M$ is an integer. In the gate voltage units, $\Delta V_{e}=$ $\left(2 E_{C}+\delta E\right) / e$ is a period of the conductance oscillations. This property reflects symmetry with respect to adding (removing) an electron to the quantum dot. Secondly, due to the electron-hole symmetry, conductance is an even function of $\Delta_{0}: G\left(\Delta_{0}\right)=G\left(-\Delta_{0}\right)$.

The linear conductance (22) as a function of the dimensionless gate voltage, $\Delta_{0}$, at $\delta E=E_{C}$ is plotted in Fig. 4 for different temperatures. At low temperatures there are sharp Coulomb blockade peaks. At high temperatures, $T \gg E_{C}, \delta E$, Coulomb blockade is lifted and small oscillations of the conductance can be observed. These oscillations are slightly non-sinusoidal and given

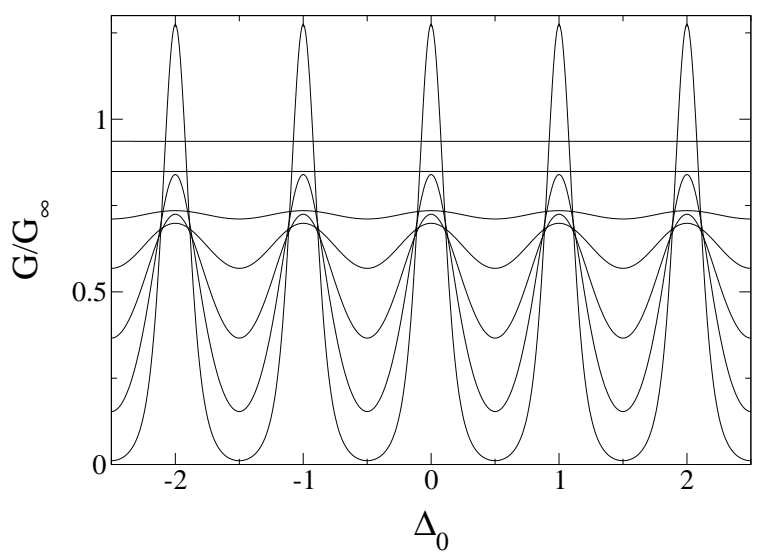

FIG. 4: Coulomb blockade oscillations of the conductance as a function of the dimensionless gate voltage, $\Delta_{0}$ at $\delta E=$ $E_{C}$. Curves are plotted for different temperatures: $T / E_{C}=$ $T / \delta E \equiv 1 / \delta=0.2,0.35,0.5,0.7,1,2,5$. 
by the following asymptotic formula:

$$
\begin{array}{r}
\frac{G\left(\Delta_{0}\right)-\overline{G\left(\Delta_{0}\right)}}{G_{\infty}}=2 \pi^{3 / 2} \frac{e^{-\varepsilon_{0} / 4}}{\sqrt{\varepsilon_{0}}}\left[e^{-\pi^{2} / \varepsilon_{0}} \cos \left(2 \pi \Delta_{0}\right)\right. \\
\left.+e^{-2 \pi^{2} / \varepsilon_{0}} \cos \left(4 \pi \Delta_{0}\right)+O\left(e^{-3 \pi^{2} / \varepsilon_{0}}\right)\right]
\end{array}
$$

where $\overline{G\left(\Delta_{0}\right)}$ is the average value of the conductance. The second term in Eq. (24) is due to inherently nonsinusoidal nature of the conductance oscillations, see Fig. 4 To derive this expression one can use Poisson's summation formula.

To study the line shape of a separate peak let us consider the limit of large charging energy: $E_{C} \gg T, \delta E$ or, equivalently, $\varepsilon_{0} \gg 1, \delta$ in the dimensionless units. In Eq. (22) only $N=-1,0$ terms in the sum over $N$ give substantial contribution to the conductance near $\Delta_{0}=0$; all other terms are exponentially suppressed. Besides, sum over $j$ at the $N=-1$ is $O\left(e^{-2 \varepsilon_{0}}\right)$ and, therefore, can also be neglected. Hence, line shape of the conductance peak at $\Delta_{0}=0$ is given by

$$
\frac{G\left(\Delta_{0}\right)}{G_{\infty}}=\frac{\delta}{1+e^{-2 \varepsilon_{0} \Delta_{0}}} \sum_{j} \frac{n_{j}}{1+e^{-j \delta+2 \varepsilon_{0} \Delta_{0}}} .
$$

It is more instructive to rewrite this equation as follows:

$$
\frac{G\left(\phi_{0}\right)}{G_{\infty}}=\frac{\delta}{1+e^{-\beta e \phi_{0}}} \sum_{j} \frac{n_{j}}{1+e^{-j \delta+\beta e \phi_{0}}},
$$

where $\phi_{0}=V_{e}-V_{e}^{(0)} ; V_{e}^{(0)}$ is chosen so that $\phi_{0}=0$ corresponds to center of the conductance peak. In the classical regime, $T \gg \delta E$, line shape of the conductance peak is given by ${ }^{7}$

$$
\frac{G\left(\phi_{0}\right)}{G_{\infty}}=\frac{\beta e \phi_{0}}{2 \sinh \left(\beta e \phi_{0}\right)} .
$$

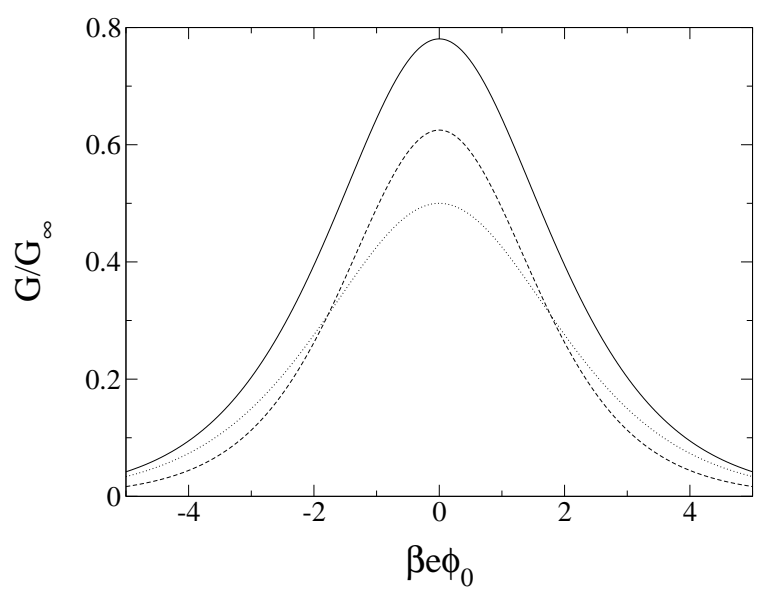

FIG. 5: The exact line shape of the conductance peak, Eq. (26), plotted at $1 / \delta \equiv T / \delta E=0.4$ (solid curve). Dotted curve corresponds to blindly applying classical regime formula (27) at $T / \delta E=0.4$. Dashed curve corresponds to blindly applying formula (28) at $T / \delta E=0.4$. The argument of the plot is linear function of the gate voltage, $\phi_{0}$.
In the opposite limit of $\delta E \gg T$ :

$$
\frac{G\left(\phi_{0}\right)}{G_{\infty}}=\frac{\delta}{2\left[1+\cosh \left(\beta e \phi_{0}\right)\right]} .
$$

The exact line shape of the conductance peak, Eq. (26), at $T / \delta E=0.4$ is shown in Fig. [5] On the same figure we also plotted two conductance peaks in the limiting cases, Eqs. (27) and (28), out of their validity region at $T / \delta E=0.4$. Nevertheless, it is interesting that the exact conductance peak is higher than both of the limiting cases peaks. The peak's height is given by

$$
\frac{G(0)}{G_{\infty}}=\frac{\delta}{2} \sum_{j} \frac{n_{j}}{1+e^{-j \delta}}=\left\{\begin{array}{ll}
1 / 2, & T \gg \delta E \\
\delta / 4, & \delta E \gg T \gg \Gamma_{i}
\end{array} .\right.
$$

Temperature dependence of the conductance peak's height was numerically calculated in the Ref. 10, see Fig. 2 there.

\section{APPLICATION TO TUNNELING THROUGH QUANTUM HALL EDGE STATES IN A QUANTUM DOT}

Formulas for the linear conductance in the case of equidistant energy levels in a dot and spinless fermions derived in the previous section can be applied to a number of physical problems.

Let us consider, for example, a quantum dot formed by confining a two-dimensional electron gas by a circularly symmetric electrostatic potential, $U(r)$. We assume that $U(r)$ is zero at the origin and takes large value at $r=R$, where $R$ is the radius of the dot, Fig. 6 6et us apply a strong magnetic field, $B$, perpendicular to the plane of the dot. This situation corresponds to the quantum Hall regime and was reviewed in Ref. 14 .

To solve the one-electron Schrödinger equation in this geometry it is convenient to choose the symmetric gauge. Then, angular momentum is, clearly, an integral of motion. In each Landau level, $n$, states with larger angu-

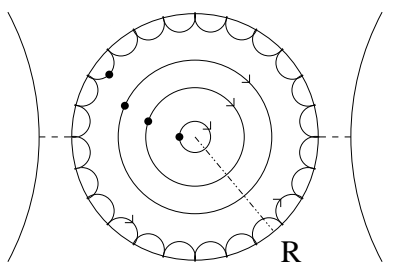

a)

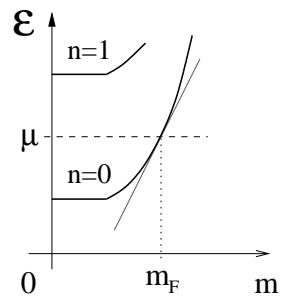

b)
FIG. 6: Geometry and spectrum of the quantum dot states: a) symmetric gauge eigenstates, $|m\rangle$, including edge state are shown schematically; b) energy spectrum of the eigenstates with different angular momenta, two lowest Landau levels are shown. 
lar momentum, $|m\rangle$, are localized further from the origin, near a circle with radius $R_{m}=l_{H} \sqrt{2(m+1)}$, where $l_{H}=\sqrt{\hbar c / e B}$ is the magnetic length, $c$ is the speed of light. The presence of the confinement potential leads to an increase in energy for the symmetric gauge eigenstates with $R_{m}$ of order or larger than $R$ (or, $m$ of order or larger than $m_{F}$, see Fig. 6b). For the states with $R_{m} \sim R$ an electron is influenced by both the electric field of the boundary, $E(R)=U^{\prime}(R) / e$, and strong, perpendicular to the electric, magnetic field. Thus, near the edge electron executes rapid cyclotron orbits centered on a point that slowly drifts in the direction of $\mathbf{E} \times \mathbf{B}$, that is, along the boundary. Thus, Quantum Hall edge states are formed, Fig. 66. It is important to notice that in this closed geometry electron system has only one edge. In this consideration we also assume that $l_{H} \ll R$.

For simplicity let us consider the case when only the zeroth Landau level crosses the chemical potential, that is, there is only one type of edge states. This corresponds to a sufficiently strong magnetic field so that filling factor, $\nu$ is equal to 1 , Fig. 6b.

Now, we are ready to consider transport through this type of quantum dot in the strong magnetic field. Let us weakly couple it to two leads and apply an infinitesimally small bias voltage between them. An electron from the left lead can now tunnel into dot's edge state and then tunnel into the right lead as illustrated by dashed lines in Fig. 6a .

The energy spectrum of edge states can be linearized as follows

$$
\mathcal{E}_{m}=\mu+\left.\frac{\partial \mathcal{E}}{\partial m}\right|_{m_{F}}\left(m-m_{F}\right) .
$$

Thus, energy levels of the edge states are equally spaced with the spacing 14

$$
\delta E=\left.\frac{\partial \mathcal{E}_{m}}{\partial m}\right|_{m_{F}}=\left.\left.\frac{\partial \mathcal{E}_{m}}{\partial R_{m}}\right|_{R} \frac{\partial R_{m}}{\partial m}\right|_{m_{F}}=e E(R) \frac{l_{H}^{2}}{R} .
$$

This fact makes formulas derived in the previous section applicable to this problem. Essential assumption here is that dispersion curve, Fig. 6b is almost linear in the range of angular momentums: $\left|m-m_{F}\right| \lesssim \Delta m$, where $\Delta m=\max (1, T / \delta E)$.

In the case at hand, spacing, $\delta E$ is inversely proportional to the size of a dot just like charging energy, $E_{C}$. Hence, their ratio does not depend on the size of a dot and is given by

$$
\frac{\delta E}{E_{C}} \sim \frac{\epsilon}{\alpha} \frac{E(R)}{B},
$$

where $\epsilon$ is the dielectric constant of the media around the interface; $\alpha=e^{2} / \hbar c$ is the fine structure constant. Therefore, in this case oscillations of the conductance given by Eq. (22) are determined by only one parameter $\delta=\delta E / T$.

In conclusion, let us consider the case of an arbitrary shaped quantum dot. In this case, $m$ is just the index of an edge state and no longer associated with the angular momentum. The phase along the boundary for the $m$-th edge state is

$$
\theta_{m}=\int_{0}^{L} d x k_{m}(x)
$$

where $k_{m}(x)$ is the corresponding wave vector, $x$ parametrizes the boundary, and $L$ is its length. The phase difference between two consecutive edge states is

$$
\theta_{m+1}-\theta_{m}=2 \pi=\int_{0}^{L} d x\left[k_{m+1}(x)-k_{m}(x)\right],
$$

where $k_{m+1}(x)-k_{m}(x)=\left(\mathcal{E}_{m+1}-\mathcal{E}_{m}\right) / \hbar v(x), v(x)=$ $c E(x) / B$ is a drift speed along the boundary. Then, the spacing between edge states' energy levels is

$$
\delta E=2 \pi \hbar\left[\int_{0}^{L} \frac{d x}{v(x)}\right]^{-1}=2 \pi e l_{H}^{2}\left[\int_{0}^{L} \frac{d x}{E(x)}\right]^{-1} .
$$

Though the electric field $E(x)$ at the boundary slightly changes as one goes from one edge state to the other, this effect is small and we neglect it. Therefore, the energy levels of the edge states are equidistant with the spacing given by Eq. (35).

In the case of the circularly symmetric quantum dot, $E(x)$ is constant, and one can easily perform the integration in Eq. (35). This leads to the previously obtained expression for the level spacing, Eq. (31).

\section{LINEAR CONDUCTANCE IN THE SPIN- $\frac{1}{2}$ CASE}

Formula (7) for the linear conductance in the spinless case can be easily generalized to the spin- $\frac{1}{2}$ case by counting each energy level twice ${ }^{10}$

$$
\begin{aligned}
G= & 2 \frac{e^{2}}{h T} \sum_{i=1}^{\infty} \frac{\Gamma_{i}^{l} \Gamma_{i}^{r}}{\Gamma_{i}^{l}+\Gamma_{i}^{r}} \sum_{N_{e}=1}^{\infty} P_{e q}\left(N_{e}\right) F_{e q}\left(E_{i \uparrow} \mid N_{e}\right) \\
& \times\left[1-n_{F}\left(E_{i}-\mu+U(N)-U(N-1)\right)\right],
\end{aligned}
$$

where $F_{e q}\left(E_{i \uparrow} \mid N_{e}\right)$ is the occupation number of the quantum dot's energy level $i$ with a spin-up electron, $(i, \uparrow)$ in the canonical ensemble: number of electrons in the dot, $N_{e}$, is fixed.

As in the spinless case, to carry out analytical consideration we have to assume that energy levels in the quantum dot are equally spaced. Presence of the spin degeneracy makes the calculations more complicated.

First of all, let us find the occupation number $F_{e q}\left(E_{i \uparrow} \mid N_{e}\right)$. Let us consider spin-up and spin-down electron subsystems. Ground state energy of the system is

$$
E_{g}=\left(N_{\uparrow}^{2}+N_{\downarrow}^{2}\right) \frac{\delta E}{2}=\left[\left(N_{\uparrow}+N_{\downarrow}\right)^{2}+\left(N_{\uparrow}-N_{\downarrow}\right)^{2}\right] \frac{\delta E}{4},
$$


where $N_{\sigma}=\left(N_{e}\right)_{\sigma}-N_{i} / 2$ is the number of excess electrons in the spin- $\sigma$ subsystem; $N_{i}$ is chosen even. $N_{\uparrow}+N_{\downarrow} \equiv N$ is the total number of excess electrons in the quantum dot; $\left(N_{\uparrow}-N_{\downarrow}\right) / 2 \equiv S_{z}$ is $z$-component of the total electron spin. Using these identities, one can find that

$$
E_{g}=\frac{1}{4} N^{2} \delta E+S_{z}^{2} \delta E .
$$

While $S_{z}$ is subjected to the thermodynamic fluctuations, $N$ is fixed.

The occupation number in question, $F_{e q}\left(E_{i \uparrow} \mid N_{e}\right) \equiv$ $n_{j \uparrow}$, is known if, in addition to $N$, the $z$-component of the total spin, $S_{z}$, is fixed. In the case of an even number of electrons, parameter $\zeta^{\prime}$ for the spin-up electron subsystem is equal to $\zeta+S_{z} \delta E$, given $S_{z}$, see Figs. 7 a and $7 \mathrm{~b}$. Occupation numbers in the spin-up subsystem at fixed $S_{z}$ are given by Eq. (9) with the appropriately chosen parameter $\zeta^{\prime}: n_{j-S_{z}}$.

However, $z$-component of the total spin, $S_{z}$, is not fixed but subjected to the thermodynamic fluctuations. Therefore, to find the occupation numbers, $n_{j \uparrow}^{e v}$, we have to account for all possible values of $S_{z}$ :

$$
n_{j \uparrow}^{e v}=\sum_{S_{z}=-N_{e} / 2}^{N_{e} / 2} P\left(S_{z}\right) n_{j-S_{z}}
$$

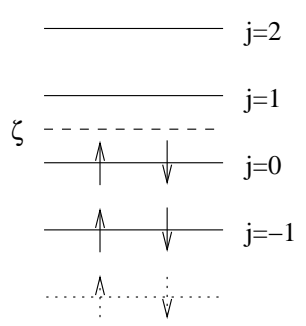

a)

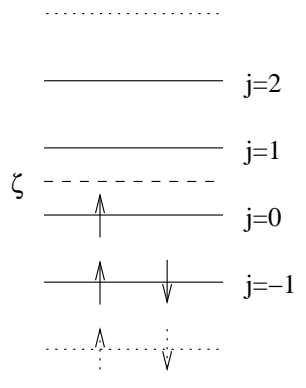

c)

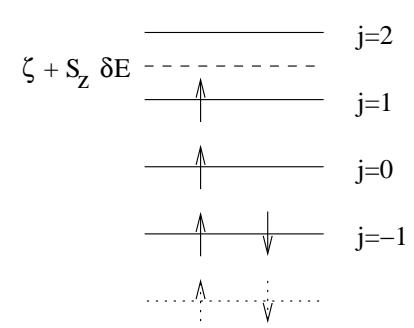

b)

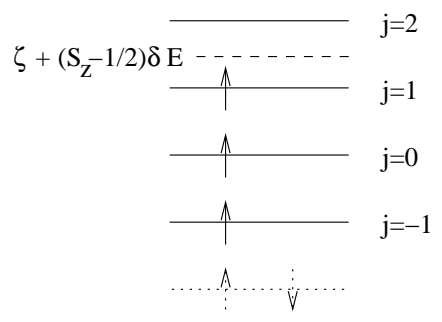

d)
FIG. 7: Parameter $\zeta^{\prime}$ of the spin-up electron subsystem for even number of electrons: a) at $S_{z}=0$, b) at arbitrary integer $S_{z}\left(S_{z}=1\right.$ case is shown); for odd number of electrons: c) at $S_{z}=1 / 2$, d) at arbitrary half-integer $S_{z}\left(S_{z}=3 / 2\right.$ case is shown). where

$$
P\left(S_{z}\right)=\frac{e^{-S_{z}^{2} \delta}}{\sum_{S_{z}=-N_{e} / 2}^{N_{e} / 2} e^{-S_{z}^{2} \delta}}
$$

is the probability that $z$-component of the total spin of the quantum dot is equal to $S_{z}$. Substituting Eqs. (39) and (9) in Eq. (38) we obtain

$$
n_{j \uparrow}^{e v}=\sum_{m=1}^{\infty}(-1)^{m-1} e^{-\left[m^{2}+(2 j-1) m\right] \frac{\delta}{2}} \frac{\sum_{S_{z}=-N_{e} / 2}^{N_{e} / 2} e^{-\left(S_{z}^{2}-m S_{z}\right) \delta}}{\sum_{S_{z}=-N_{e} / 2}^{N_{e} / 2} e^{-S_{z}^{2} \delta}} .
$$

Since number of electrons in the quantum dot is even, $S_{z}$ may take only integer values. Therefore,

$$
n_{j \uparrow}^{e v}=\sum_{m=1}^{\infty}(-1)^{m-1} e^{-\left[\frac{m^{2}}{4}+\left(j-\frac{1}{2}\right) m\right] \delta} \frac{\sum_{S_{z}=-\infty}^{\infty} e^{-\left(S_{z}-\frac{m}{2}\right)^{2} \delta}}{\sum_{S_{z}=-\infty}^{\infty} e^{-S_{z}^{2} \delta}},
$$

where we extended limits of the sum over $S_{z}$ to infinities since the Fermi energy is the largest energy scale in the problem. Separating $m=2 r-1$ and $m=2 r$ parts of the sum, where $r$ is a positive integer, we obtain final expression for the occupation numbers in the case of even number of electrons:

$$
n_{j \uparrow}^{e v}=A(\delta) \sum_{r=1}^{\infty} e^{-\left(r-\frac{1}{2}\right)\left(r+2 j-\frac{3}{2}\right) \delta}-\sum_{r=1}^{\infty} e^{-r(r+2 j-1) \delta},
$$

where

$$
A(\delta)=\frac{\sum_{s=-\infty}^{\infty} e^{-\left(s-\frac{1}{2}\right)^{2} \delta}}{\sum_{s=-\infty}^{\infty} e^{-s^{2} \delta}}
$$

In two limiting cases

$$
A(\delta)=\left\{\begin{array}{l}
2 e^{-\delta / 4}\left[1+O\left(e^{-\delta}\right)\right], \quad \delta E \gg T \\
1-4 e^{-\pi^{2} / \delta}+O\left(e^{-2 \pi^{2} / \delta}\right), \quad T \gg \delta E
\end{array} .\right.
$$

Analytical expression for the high-temperature limit of $A(\delta)$ can be obtained using Poisson's summation formula.

One can easily prove the following properties of the occupation numbers $n_{j \uparrow}^{e v}$ valid at arbitrary temperature:

$$
\begin{aligned}
n_{j \uparrow}^{e v} & =1-n_{1-j, \uparrow}^{e v}, \\
e^{j \delta} n_{j \uparrow}^{e v}+e^{-j \delta} n_{-j \uparrow}^{e v} & =A(\delta) e^{\delta / 4} .
\end{aligned}
$$

They are valid due to the electron-hole symmetry and similar to the following properties of the Fermi-Dirac 
distribution: $n_{F}(E)=1-n_{F}(-E)$ and $e^{\beta E} n_{F}(E)+$ $e^{-\beta E} n_{F}(-E)=1$.

Similarly, one can find occupation numbers in the case of odd number of electrons in the quantum dot, $N_{e}$. Energy level which contains one electron at $T=0$ will be referred to as $j=0$ level. In this case electron-hole symmetry corresponds to $j \rightarrow-j$ transformation. Parameter $\zeta^{\prime}$ of the spin-up electron subsystem at a given $S_{z}$ is equal to $\zeta+\left(S_{z}-1 / 2\right) \delta E$, see Figs. 7 r and 7 . Therefore,

$$
\begin{array}{r}
n_{j \uparrow}^{o d}=\sum_{S_{z}=-N_{e} / 2}^{N_{e} / 2} P\left(S_{z}\right) n_{j-\left(S_{z}-\frac{1}{2}\right)} \\
=\sum_{m=1}^{\infty}(-1)^{m-1} e^{-\left(\frac{m^{2}}{4}+j m\right) \delta} \frac{\sum_{S_{z}=-N_{e} / 2}^{N_{e} / 2} e^{-\left(S_{z}-\frac{m}{2}\right)^{2} \delta}}{\sum_{S_{z}=-N_{e} / 2}^{N_{e} / 2} e^{-S_{z}^{2} \delta}} .
\end{array}
$$

Since number of electrons in the quantum dot is odd, $S_{z}$ may take only half-integer values. Separating odd and even parts of the sum over $m$, we obtain:

$$
n_{j \uparrow}^{o d}=\frac{1}{A(\delta)} \sum_{r=1}^{\infty} e^{-\left(r-\frac{1}{2}\right)\left(r+2 j-\frac{1}{2}\right) \delta}-\sum_{r=1}^{\infty} e^{-r(r+2 j) \delta} .
$$

Property of the electron-hole symmetry reads as follows: $n_{j \uparrow}^{o d}=1-n_{-j \uparrow}^{o d}$.

It turns out that there exists simple relation between $n^{e v}$ and $n^{o d}$ occupation numbers:

$$
n_{j \uparrow}^{e v}=A(\delta) e^{-\left(j-\frac{1}{4}\right) \delta} n_{-j \uparrow}^{o d} .
$$

This property is the analog of $n_{F}(E)=e^{-\beta E} n_{F}(-E)$ one of the Fermi-Dirac distribution. It will allow us to get rid of $n^{\text {od }}$ occupation numbers in the final expression for the conductance.

Now we are in a position to find the probability that a dot, in thermodynamic equilibrium with the reservoirs, contains $N_{e}$ electrons, $P_{e q}\left(N_{e}\right)$. Equation (13) written for the spinless case is still applicable if we keep in mind that energy levels in the quantum dot are doubly degenerate. Partition function of the dot's internal degrees of freedom in the canonical ensemble is

$$
\begin{aligned}
\mathcal{Z}\left(N_{e}\right) & =\sum_{\left\{n_{i \sigma}\right\}} e^{-\beta \sum\left(E_{i}-\mu\right) n_{i \sigma}} \delta_{N_{e}, \sum n_{i \sigma}} \\
& =\sum_{N_{e}^{\uparrow}=0}^{N_{e}} \sum_{N_{e}^{\downarrow}=0}^{N_{e}} Z\left(N_{e}^{\uparrow}\right) Z\left(N_{e}^{\downarrow}\right) \delta_{N_{e}, N_{e}^{\uparrow}+N_{e}^{\downarrow}},
\end{aligned}
$$

where

$$
Z\left(N_{e}^{\uparrow}\right)=\sum_{\left\{n_{i}\right\}} e^{-\beta \sum\left(E_{i}-\mu\right) n_{i}} \delta_{N_{e}^{\uparrow}, \sum n_{i}}
$$

is the partition function of the spin-up electron subsystem in the canonical ensemble. Mathematically, expression for $Z\left(N_{e}^{\uparrow}\right)$ is identical to the one for $Z\left(N_{e}\right)$ in the spinless case. Thus, one can directly apply the result obtained previously, Eq. (14):

$$
Z\left(N_{e}^{\uparrow}\right)=e^{-\left(N_{e}^{\uparrow}-N_{i} / 2\right)^{2} \delta / 2} Z_{e x c},
$$

where $N_{e}^{\uparrow}=N_{i} / 2$ is the equilibrium number of electrons in the spin-up subsystem. Similar result is valid for the partition function of the spin-down electron subsystem, $Z\left(N_{e}^{\downarrow}\right)$. Substituting these results in Eq. (47) we obtain:

$$
\begin{array}{r}
\mathcal{Z}\left(N_{e}\right)=Z_{\text {exc }}^{2} \\
\times \sum_{N_{e}^{\uparrow}=0}^{N_{e}} \sum_{N_{e}^{\downarrow}=0}^{N_{e}} e^{-\left[\left(N_{e}^{\uparrow}-N_{i} / 2\right)^{2}+\left(N_{e}^{\downarrow}-N_{i} / 2\right)^{2}\right] \delta / 2} \delta_{N_{e}^{\uparrow}+N_{e}^{\downarrow}, N_{e}} .
\end{array}
$$

The exponent can be simplified as follows

$$
\left(N_{e}^{\uparrow}-\frac{N_{i}}{2}\right)^{2}+\left(N_{e}^{\downarrow}-\frac{N_{i}}{2}\right)^{2}=\frac{N^{2}}{2}+\frac{\left(N_{e}^{\uparrow}-N_{e}^{\downarrow}\right)^{2}}{2},
$$

hence,

$$
\begin{gathered}
\frac{\mathcal{Z}\left(N_{e}\right)}{Z_{\text {exc }}^{2}}=e^{-N^{2} \delta / 4} \sum_{N_{e}^{\uparrow}=0}^{N_{e}} \sum_{N_{e}^{\downarrow}=0}^{N_{e}} e^{-\left(N_{e}^{\uparrow}-N_{e}^{\downarrow}\right)^{2} \delta / 4} \delta_{N_{e}^{\uparrow}+N_{e}^{\downarrow}, N_{e}} \\
=e^{-N^{2} \delta / 4} \sum_{N_{e}^{\uparrow}=0}^{N_{e}} e^{-\left(N_{e}^{\uparrow}-N_{e} / 2\right)^{2} \delta}=e^{-N^{2} \delta / 4} \sum_{s=-N_{e} / 2}^{N_{e} / 2} e^{-s^{2} \delta},
\end{gathered}
$$

where in the second equality we took advantage of the delta symbol. Sum in the last line is taken over integer values of $s$ if $N_{e}$ is even or half-integer values of $s$ if $N_{e}$ is odd. Limits of the sum over $s$ can be extended to infinities since we assume that $\mu \gg T$. According to Eq. (13) probability that quantum dot, in thermodynamic equilibrium with the reservoirs, contains $N_{e}$ electrons is

$$
\begin{gathered}
P_{e q}\left(N_{e}\right)=\frac{\mathcal{Z}\left(N_{e}\right)}{Z_{\mu}} e^{-\beta U(N)}=\frac{Z_{e x c}^{2}}{Z_{\mu}} e^{-\beta \tilde{U}(N)} \sum_{s=-N_{e} / 2}^{N_{e} / 2} e^{-s^{2} \delta} \\
=\frac{e^{-\beta \tilde{U}(N)} \sum_{s=-N_{e} / 2}^{N_{e} / 2} e^{-s^{2} \delta}}{\sum_{N=e v} e^{-\beta \tilde{U}(N)} \sum_{s} e^{-s^{2} \delta}+\sum_{N=o d} e^{-\beta \tilde{U}(N)} \sum_{s} e^{-\left(s-\frac{1}{2}\right)^{2} \delta}},
\end{gathered}
$$

where $\tilde{U}(N) \equiv U(N)+N^{2} \delta E / 4$; and we used the fact that $N$ and $N_{e}$ have the same parity since $N_{i}$ is chosen even. Therefore, sums over $N=e v$ and $N=o d$ are taken over $N=0, \pm 2, \pm 4, \ldots$ and $N= \pm 1, \pm 3, \ldots$ values, respectively. At this point in the calculation we need to specify whether the total number of electrons in the dot is even or odd:

$$
P_{e q}\left(N_{e}\right)=\left\{\begin{array}{l}
\left(D^{\prime}\right)^{-1} e^{-\beta \tilde{U}(N)}, \quad N \text { is even } \\
\left(D^{\prime}\right)^{-1} A(\delta) e^{-\beta \tilde{U}(N)}, \quad N \text { is odd }
\end{array},\right.
$$

where

$$
D^{\prime} \equiv \sum_{N=\text { even }} e^{-\beta \tilde{U}(N)}+A(\delta) \sum_{N=\text { odd }} e^{-\beta \tilde{U}(N)}
$$


Now we are prepared to calculate the conductance, Eq. (36), in the case of the equidistant double degenerate energy levels in the dot at an arbitrary $\delta E / T$ and $E_{C} / \delta E$ ratios. Similarly to the consideration in the spinless case we assume that quantum dot is weakly coupled to the leads via multichannel tunnel junctions, and tunneling widths of the energy levels in the quantum dot, $\Gamma_{i}^{l}$ and $\Gamma_{i}^{r}$, are slowly changing functions of the energy, $E_{i}$. Then, these tunneling widths can be considered constants and evaluated at the chemical potential: $\Gamma_{i}^{l} \approx \Gamma_{\mu}^{l} \equiv \Gamma^{l}$; $\Gamma_{i}^{r} \approx \Gamma_{\mu}^{r} \equiv \Gamma^{r}$. Furthermore, they can be expressed via conductances of the corresponding junctions:

$$
\Gamma^{l}=\frac{h G^{l}}{e^{2}} \frac{\delta E}{2}, \quad \Gamma^{r}=\frac{h G^{r}}{e^{2}} \frac{\delta E}{2} .
$$

There is an additional factor of $1 / 2$ here compared to the spinless case, Eq. (17), due to the double degeneracy of each energy level in the quantum dot. First of all, let us break the sum over $N_{e}$ in Eq. (36) in two parts: $N=$ even and $N=o d d$, and apply Eqs. (152) and (150):

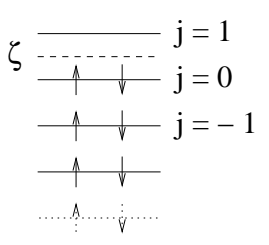

a)

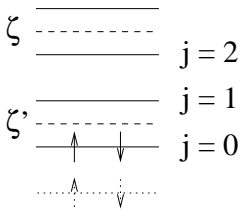

b)

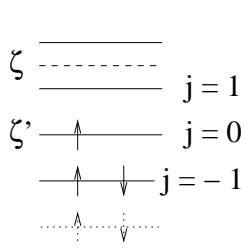

c)
FIG. 8: Mapping of the sum over $i$ onto the sum over $j$ for a) $N=0$; b) even $N(N=-4$ case is shown); c) odd $N$ ( $N=-3$ case is shown).

$$
\begin{aligned}
G= & \frac{G^{l} G^{r}}{G^{l}+G^{r}} \frac{\delta}{D^{\prime}}\left(\sum_{N=\text { even }} e^{-\beta \tilde{U}(N)} \sum_{i=1}^{\infty} F_{e q}\left(E_{i \uparrow} \mid N_{e}\right)\left\{1-n_{F}\left[E_{i}-\mu+U(N)-U(N-1)\right]\right\}\right. \\
& \left.+A(\delta) \sum_{N=\text { odd }} e^{-\beta \tilde{U}(N)} \sum_{i=1}^{\infty} F_{e q}\left(E_{i \uparrow} \mid N_{e}\right)\left\{1-n_{F}\left[E_{i}-\mu+U(N)-U(N-1)\right]\right\}\right) .
\end{aligned}
$$

To take advantage of the occupation numbers we derived, Eqs. (40) and (45), we need to map each of the sums over $i$ onto the sum over $j$. The first sum over $i$ in Eq. (53.) is taken at even number of excess electrons, see Figs. 8 a and $8 \mathrm{~b}$, hence

$$
\begin{aligned}
F_{e q}\left(E_{i \uparrow} \mid N_{e}\right) & =n_{j \uparrow}^{e v} \\
E_{i}-\mu=E_{i}-\zeta^{\prime}+\left(\zeta^{\prime}-\zeta\right) & =\left(j-\frac{1}{2}\right) \delta E+\frac{1}{2} N \delta E .
\end{aligned}
$$

Remember that by properly choosing "zero" of the gate voltage we put $\zeta=\mu$. The second sum over $i$ is taken at odd number of excess electrons, see Figs. 8 a and 8 ; therefore

$$
\begin{aligned}
F_{e q}\left(E_{i \uparrow} \mid N_{e}\right) & =n_{j \uparrow}^{o d}, \\
E_{i}-\mu=E_{i}-\zeta^{\prime}+\left(\zeta^{\prime}-\zeta\right) & =j \delta E+\frac{1}{2} N \delta E .
\end{aligned}
$$

We will also use the following identities:

$$
\tilde{U}(N)=\left(E_{C}+\frac{\delta E}{4}\right) N^{2}-e V_{e} N
$$

$$
=\left(E_{C}+\frac{\delta E}{4}\right)\left(N-\Delta+\frac{1}{2}\right)^{2}+\mathcal{C}_{2},
$$

where $\mathcal{C}_{2}=-\left(e V_{e}\right)^{2} /\left(4 E_{C}+\delta E\right)$;

$$
\Delta \equiv \frac{e V_{e}}{2\left(E_{C}+\delta E / 4\right)}+\frac{1}{2}
$$

is the dimensionless gate voltage, $\Delta=0$ corresponds to a position of the conductance peak; and

$$
\begin{aligned}
& U(N)-U(N-1)=(2 N-1) E_{C}-e V_{e} \\
& =(2 N-1) E_{C}-2\left(E_{C}+\frac{\delta E}{4}\right)\left(\Delta-\frac{1}{2}\right) .
\end{aligned}
$$

Substituting these results in Eq. (53) we obtain

$$
\frac{G(\Delta)}{G_{\infty}}=\frac{\delta}{D} \sum_{N=\text { even }} e^{-\varepsilon(N-\Delta+1 / 2)^{2}} \sum_{j=-\infty}^{\infty} n_{j \uparrow}^{e v}\left[\frac{1}{e^{-(j-1 / 4) \delta-2(N-\Delta) \varepsilon}+1}+\frac{1}{e^{-(j-1 / 4) \delta+2(N+1-\Delta) \varepsilon}+1}\right]
$$


where

$$
D=\exp \left(\beta \mathcal{C}_{2}\right) D^{\prime}=\sum_{N=\text { even }} e^{-\varepsilon(N-\Delta+1 / 2)^{2}}+A(\delta) \sum_{N=\text { odd }} e^{-\varepsilon(N-\Delta+1 / 2)^{2}} ;
$$

$$
\varepsilon \equiv \beta\left(E_{C}+\frac{\delta E}{4}\right)
$$

$G_{\infty} \equiv G^{l} G^{r} /\left(G^{l}+G^{r}\right)$ is the high-temperature, $E_{C}, \delta E \ll T \ll \mu$, limit of the conductance. To eliminate $n^{\text {od }}$ from the final expression we used useful property of the occupation numbers given by Eq. (46).

Formula (55) is the main result of this paper. It is the analytical expression for the linear conductance in the spin- $\frac{1}{2}$ case for equidistant energy levels in the quantum dot. One can use formula (55) to plot Coulomb blockade oscillations of the conductance as a function of the dimensionless gate voltage, $\Delta$, at arbitrary values of $E_{C}$, $\delta E$ and $T$. Particularly, when all energy scales are of the same order: $E_{C} \sim \delta E \sim T$, numerical calculation is a breeze, Fig. 9

One can immediately notice the following properties of the linear conductance. First of all, $G(\Delta)=G(\Delta+$ $2 M)$, where $M$ is an integer. In other words, $\left(4 E_{C}+\right.$ $\delta E) / e$ is the conductance period in the gate voltage units. This property reflects symmetry with respect to adding (removing) two electrons to the quantum dot. Secondly, conductance is a symmetric function with respect to the center of a valley, $\Delta^{\prime}=M+1 / 2$, where $M$ is an integer. That is, $G\left(\Delta-\Delta^{\prime}\right)=G\left(\Delta^{\prime}-\Delta\right)$. This is a reflection of the electron-hole symmetry. These properties of the conductance oscillations are not generic. They are valid due to the assumption of equally spaced energy levels in a quantum dot.

At high temperatures, $T \gg E_{C}, \delta E$, conductance peaks overlap and their maximums become almost

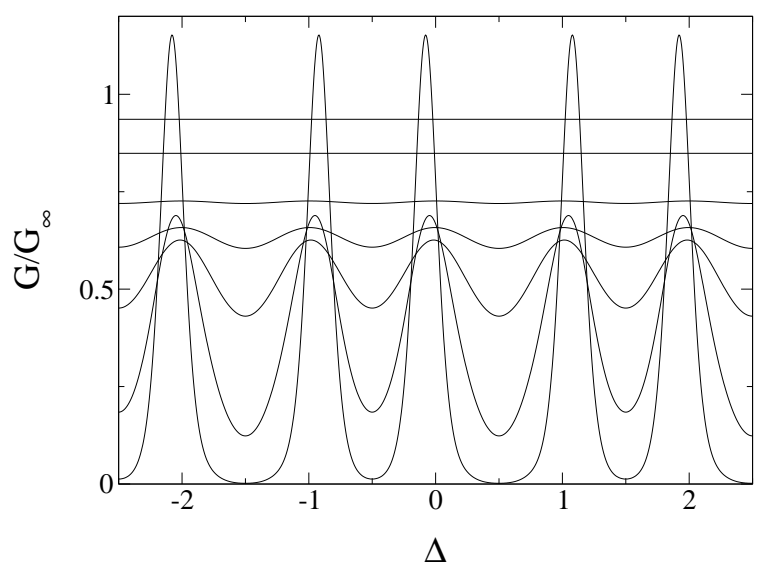

FIG. 9: Linear conductance oscillations as a function of the dimensionless gate voltage, $\Delta$, at $\delta E=E_{C}$. Curves are plotted for different temperatures: $T / E_{C}=T / \delta E \equiv 1 / \delta=$ 0.15, 0.3, 0.5, 0.7, 1, 2, 5 using Eq. (55). equidistant, Fig. 9] As a result, instead of separate peaks, the conductance in this limit has oscillatory behavior.

Let us find temperature dependence of the conductance in the valleys. In the sequential tunneling approximation the conductance in the valleys decays exponentially as $T \rightarrow 0$, Fig. [10] At low temperature number of electrons in a dot in the valleys is almost quantized. We will call the valley "odd" ("even") if it corresponds to odd (even) number of electrons in the dot. We find that at any temperature the conductance in the odd valley is larger than that in the even one, Fig. 10 This feature is robust with respect to the distribution of energy levels in a quantum dot.

However, it is important to mention that at low temperatures, $T<T_{i n}$, where

$$
T_{i n} \simeq \frac{E_{C}}{\ln \left(\frac{e^{2} / \hbar}{G^{l}+G^{r}}\right)}
$$

cotunneling 15 will dominate sequential tunneling contribution to the conductance in the valleys. Therefore, temperature dependence of the conductance in the valleys, Fig. 10] is valid only for the temperatures $T>T_{i n}$.

Let us analyze the limit of large charging energy: $E_{C} \gg T, \delta E$ or, equivalently, $\varepsilon \gg 1, \delta$ in the dimensionless units. In this limit, two adjacent peaks in the conductance have exponentially small, $\sim e^{-E_{C} / T}$, overlap with each other. Thus, it makes perfect sense to study the line shape of a separate peak. Let us determine line shape of the conductance peak near $\Delta=0$. In the numerator of Eq. (55) only the $N=0$ term in the sum over $N$ survives; moreover, at $N=0$ second term in

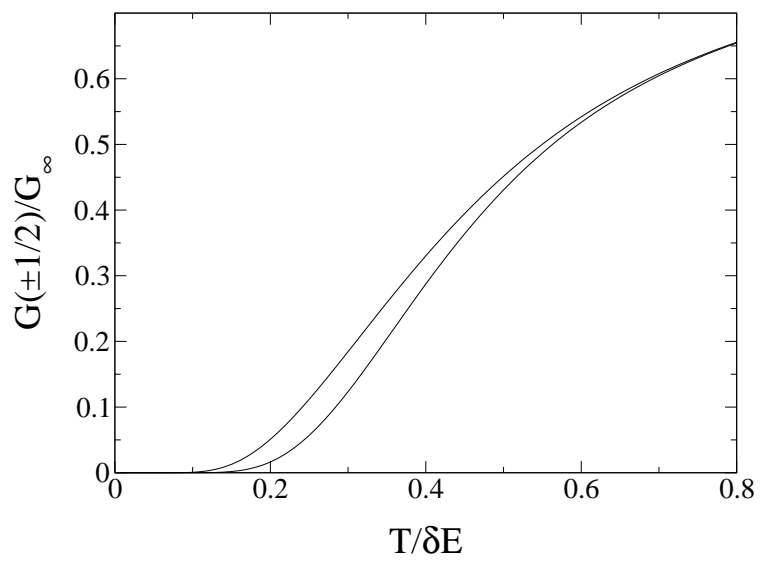

FIG. 10: Temperature dependence of the conductance in the odd, $G(-1 / 2) / G_{\infty}$ and even, $G(1 / 2) / G_{\infty}$ valleys at $\delta E=E_{C}$. Upper curve corresponds to the conductance in the odd valley. 
square brackets is $O\left(e^{-2 \varepsilon}\right)$. In the denominator, only the $N=-1,0$ terms matter. Hence, the line shape of the conductance peak near $\Delta=0$ at arbitrary $\delta E / T$ ratio is given by

$$
\frac{G(\phi)}{G_{\infty}}=\frac{\delta}{1+A(\delta) e^{-\beta e \phi}} \sum_{j=-\infty}^{\infty} \frac{n_{j \uparrow}^{e v}}{1+e^{-(j-1 / 4) \delta+\beta e \phi}}
$$

where we used the following identity:

$$
2 \varepsilon \Delta=\beta e\left(V_{e}-V_{e}^{(0)}\right)=\beta e \phi .
$$

Clearly, $\phi=0$ corresponds to $\Delta=0$. In the classical regime, $T \gg \delta E$, the line shape of the conductance peak is given by ${ }^{7}$

$$
\frac{G(\phi)}{G_{\infty}}=\frac{\beta e \phi}{2 \sinh (\beta e \phi)} .
$$

Formally, this equation is identical to that of the spinless case, Eq. (27). Nonetheless, the values of $G_{\infty}$ are different in these two cases by a factor of 2 . This is due to spin degeneracy of each energy level in the spin- $\frac{1}{2}$ case, compare Eqs. (17) and (52). In the limit of $\delta E \gg T: \underline{\underline{B}}$

$$
\frac{G(\phi)}{G_{\infty}}=\frac{\delta}{3+2 \sqrt{2} \cosh \left(\beta e \phi+\frac{1}{4} \delta-\frac{1}{2} \ln 2\right)} .
$$

This peak has its maximum at

$$
e \phi_{L T} \equiv e \phi_{m}(T \ll \delta E)=-\frac{1}{4} \delta E+\frac{\ln 2}{2} T,
$$

and is symmetric with respect to this value: $G(\phi-$ $\left.\phi_{L T}\right)=G\left(\phi_{L T}-\phi\right)$. The exact line shape of the conductance peak, Eq. (59), at $T / \delta E=0.4$ and two limiting cases conductance peaks, Eqs. (60) and (61), plotted

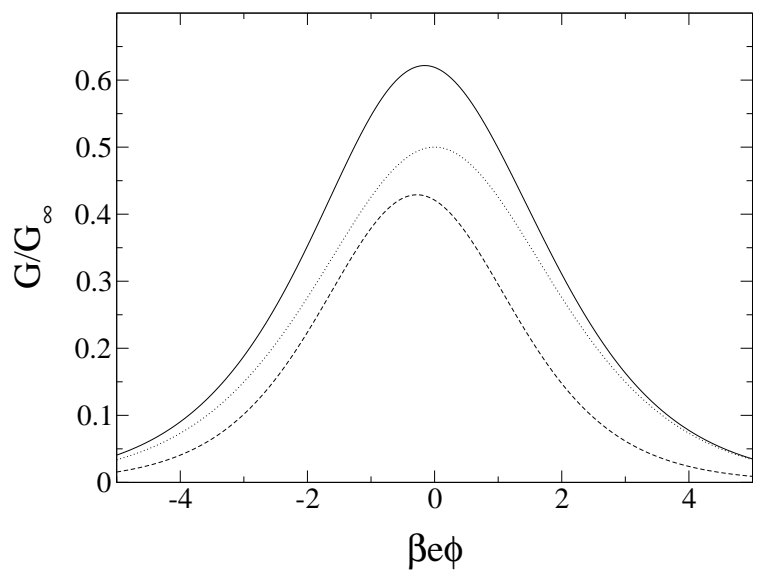

FIG. 11: The exact line shape of the conductance peak, Eq. (59), plotted at $1 / \delta \equiv T / \delta E=0.4$ (solid curve). Dotted curve corresponds to blindly applying classical regime formula, Eq. (60), at $T / \delta E=0.4$. Dashed curve corresponds to blindly applying formula (61) at $T / \delta E=0.4$. The argument of the plot is linear function of the gate voltage, $\phi$. out of their validity region at $T / \delta E=0.4$ are shown in Fig. 11 As in the spinless case, the exact conductance peak is higher than both of the limiting cases peaks.

The position of the peak's maximum, $\phi_{m}=\phi_{m}(T)$, is shifted to the left from its high temperature limit, $\phi_{C L} \equiv \phi_{m}(T \gg \delta E)=0$. It is determined by the equation for $\phi_{m}$ : $G^{\prime}\left(\phi_{m}\right)=0$, where $G(\phi)$ is given by Eq. (59). The dimensionless position of the peak's maximum, $e \phi_{m}(T) / \delta E$, as a function of the temperature, $T / \delta E$, is numerically plotted in Fig. 12

The conductance peak height is $G_{\max }=G\left(\phi_{m}\right)$. In the limiting cases:

$$
\frac{G_{\max }}{G_{\infty}}=\frac{G\left(\phi_{m}\right)}{G_{\infty}}=\left\{\begin{array}{l}
1 / 2, T \gg \delta E \\
(3-2 \sqrt{2}) \delta, \quad \delta E \gg T \gg \Gamma_{i}
\end{array} .\right.
$$

Peak's height as a function of the temperature, $T / \delta E$, can be plotted numerically, Fig. 13

\section{CONCLUSIONS}

We have studied Coulomb blockade oscillations of the linear conductance through a quantum dot weakly coupled to the leads via multichannel tunnel junctions in the sequential tunneling approximation. To obtain analytical results we have assumed that the energy levels in the dot are equally spaced. The electron-electron interaction in a quantum dot has been described by the constant interaction model; though, thermal excitations with all possible spins have been taken into account.

The linear conductance in the spinless case is given by Eq. (22). It is valid at arbitrary values of $E_{C}, \delta E$ and $T$. The line shape of an individual conductance peak at arbitrary ratio $\delta=\delta E / T$ is given by Eq. (26). Exact conductance peak is higher than both of the limiting cases peaks at any gate voltage as is illustrated in Fig [5] An analytical expression for the height of the conductance peak at any ratio $\delta$ is obtained, Eq. (29).

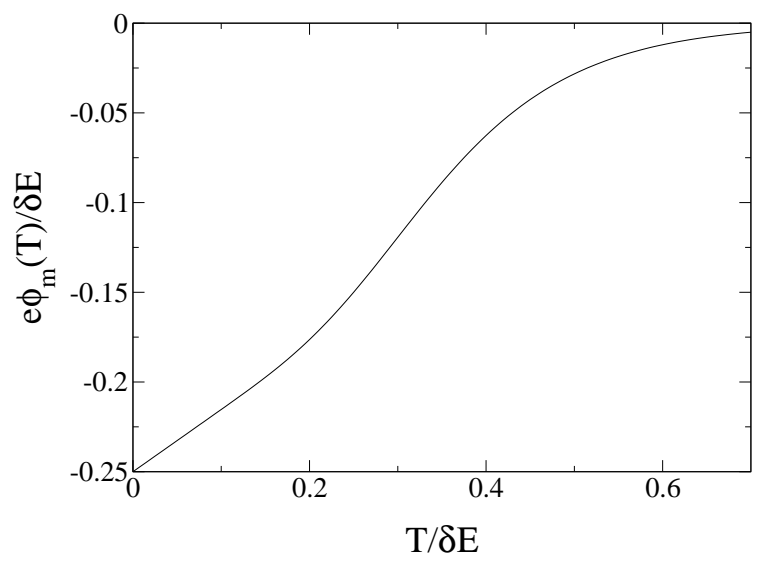

FIG. 12: Temperature dependence of the dimensionless peak's maximum position, $e \phi_{m}(T) / \delta E$. In the low temperature limit: $e \phi_{m}(0) / \delta E=-1 / 4$ according to Eq. [62]. 
In Sec. IV we applied the spinless case theory result to the problem of the transport via a dot in the quantum Hall regime. Energy levels in a dot in this case are equidistant with the spacing given by Eq. (35).

Linear conductance in the case of spin- $\frac{1}{2}$ electrons at arbitrary values of $E_{C}, \delta E$, and $T$ is given by Eq. (55). In particular, this equation allows one to plot the conductance oscillations in the regime when the charging energy, level spacing in the dot, and the temperature are all of the same order, Fig. 9. We find that the period of Coulomb blockade oscillations is doubled compared to the model with a continuous electronic spectrum in the dot. Equation (55) is the main result of the paper.

We also find that conductance in the odd valley is larger than that in the even one at any temperature, Fig. 10] The difference between conductances has the largest value at $T \approx 0.3 \delta E\left(\right.$ at $\left.\delta E=E_{C}\right)$. The sign of the difference is the same as for the quantum dot in the Kondo regime 15 Kondo effect takes place at very low temperatures, $T \lesssim T_{K} \ll T_{i n}$, where $T_{K}$ is the Kondo temperature, and leads to the logarithmic enhancement of the conductance in the odd valley 16 Our consideration shows that even-odd asymmetry exists at much higher temperatures.

Line shape of the conductance peak is given by Eq. (59). As in the spinless case, the conductance peak is higher than both of the limiting cases peaks at any gate voltage, Fig [1] As we increase the temperature peaks' maximums shift and become more equidistant, Fig 12 The peak's height as a function of the temperature is calculated numerically and plotted in Fig. [13]

Though we have found physical system which has equidistant energy levels in the spinless case, see Section IV, we are not aware of any such system in the spin- $\frac{1}{2}$ case. In the case of a chaotic quantum dot Wigner-Dyson model gives a fairly good approximation for the distribution of the energy levels of the dot. If we had assumed Wigner-Dyson distribution of the quantum dot's energy

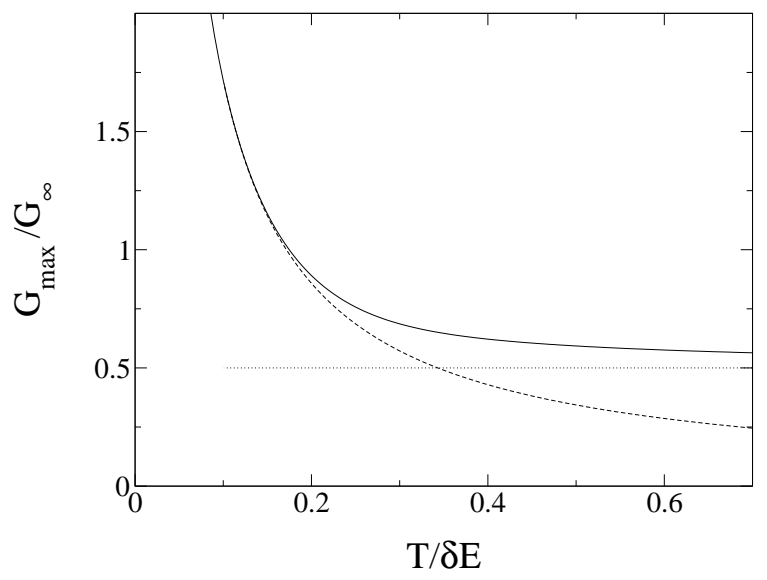

FIG. 13: Height of the conductance peak, $G_{\max } / G_{\infty}$, as a function of the temperature, $T / \delta E$. Dashed curves correspond to two limiting cases peak heights, Eq. (63). levels then we would have had to give up the hope of finding a solution. It goes back to the very difficult problem of finding occupation numbers of the dot's energy levels in the canonical ensemble. The only way to solve it is to assume that energy levels in the quantum dot are equally spaced. Then one can use the bosonization technique to find the occupation numbers. Assumption of the equidistant energy levels is in line with the level repulsion property of the Wigner-Dyson distribution. Therefore, the analytical consideration of this reasonably simplified model, in our opinion, is a significant step forward in the solution of the general problem.

Though we do not expect our quantitative results to precisely describe a quantum dot with random energy levels, they certainly give correct order of magnitude for the conductance oscillations and their generic features.

\section{Acknowledgments}

The author is greatly indebted to Prof. K.A. Matveev for drawing his attention to the problem and most stimulating discussions. The author is grateful to Prof. A. Bezryadin for helpful and stimulating discussions; Prof. H.U. Baranger for valuable comments on the manuscript and his support; and Prof. S.W. Teitsworth for helpful comments on the manuscript. This work was supported in part by the NSF Grants DMR-9974435 and DMR-0103003.

\section{APPENDIX: OCCUPATION NUMBERS IN THE CANONICAL ENSEMBLE}

In the canonical ensemble, where the total number of particles, $N_{e}$, is fixed, it is difficult to calculate fermionic occupation numbers, Eq. (8), directly. This is true even in the case of equidistant energy levels separation.

Fortunately, the bosonization technique allows one to express fermionic field annihilation and creation operators, $\psi$ and $\psi^{\dagger}$, in terms of the bosonic annihilation and creation, $a_{q}$ 's and $a_{q}^{\dagger}$ 's, and ladder, $U$ and $U^{\dagger}$, operators 13

$$
\psi^{\dagger}(x)=\frac{1}{\sqrt{L}} e^{-i k_{F} x} e^{-i \chi^{\dagger}(x)} U^{\dagger} e^{-i \chi(x)},
$$

where

$$
\chi^{\dagger}(x)=\frac{\pi x}{L} N+i \sum_{q>0} \sqrt{\frac{2 \pi}{q L}} e^{-i q x} a_{q}^{\dagger} ;
$$

$L$ is the length of the artificial system; $k_{F}$ is the Fermi wave vector; $N=N_{e}-N_{i}$ is the number of excess electrons operator; $q$ is the wave vector; and $x$ is the coordinate. Since these bosons naturally exist in the grand canonical ensemble, one can fix total number of excess electrons, $N$, and calculate occupation numbers in the bosonic basis, Eq. (9). 
Occupation numbers, Eq. (9), have the following properties:

$$
n_{j}=1-n_{1-j}, \quad n_{j} e^{j \delta / 2}=n_{-j} e^{-j \delta / 2} .
$$

They are similar to $n_{F}(E)=1-n_{F}(-E)$ and $n_{F}(E) e^{\beta E / 2}=n_{F}(-E) e^{-\beta E / 2}$ ones of the Fermi-Dirac distribution and reflect the electron-hole symmetry. Combining these two properties one can get the recursion relation:

$$
n_{j+1}=1-e^{j \delta} n_{j} .
$$

In the limit of low temperature, $\delta E \gg T$, for $j>0$ we obtain

$$
n_{j}=e^{-j \delta}-e^{-(2 j+1) \delta}+O\left[e^{-(3 j+3) \delta}\right] ;
$$

in the high temperature limit, $T \gg \delta E$ :

$$
n_{j}=\frac{1}{e^{\left(j-\frac{1}{2}\right) \delta}+1}-\frac{\delta}{8} \frac{\sinh \left[\left(j-\frac{1}{2}\right) \frac{\delta}{2}\right]}{\cosh ^{3}\left[\left(j-\frac{1}{2}\right) \frac{\delta}{2}\right]}+O\left(\delta^{2}\right)
$$

thus, we find first correction to the Fermi-Dirac distribution. The form of the correction remains valid even for the slightly non-equidistant energy levels in the dot. If this is the case we need to define ratio $\delta$ in the last expression as $\delta=\overline{\delta E} / T$, where $\overline{\delta E}$ is the mean level spacing.
1 J. von Delft, D.C. Ralph, Phys. Rep. 345, 61 (2001).

2 L.P. Kouwenhoven, C.M. Marcus, P.L. McEuen, S. Tarucha, R.M. Westervelt, and N.S. Wingreen, in Mesoscopic Electron Transport, edited by L.L. Sohn, L.P. Kouwenhoven, and G. Schön, NATO ASI, Ser. E, Vol. 345 (Kluwer, Dordrecht, 1997), pp. 105-214.

3 D. Porath, O. Millo, J. Appl. Phys. 81, 2241 (1997); D. Porath, Y. Levi, M. Tarabiah, and O. Millo, Phys. Rev. B 56, 9829 (1997).

${ }^{4}$ H. Park, J. Park, A.K.L. Lim, E.H. Anderson, A.P. Alivisatos, and P.L. McEuen, Nature 407, 57 (2000).

5 Y. Ji, M. Heiblum, D. Sprinzak, D. Mahalu, H. Shtrikman, Science 290, 779 (2000).

6 M. Bockrath, D.H. Cobden, P.L. McEuen, N.G. Chopra, A. Zettl, A. Thess, R.E. Smalley, Science 275, 1922 (1997).

7 L.I. Glazman, R.I. Shekhter, J. Phys. Condens. Matter 1, 5811 (1989).

${ }^{8}$ L.I. Glazman, K.A. Matveev, Pis'ma Zh. Eksp. Teor. Fiz. 48, 403 (1988) [JETP Lett. 48, 445 (1988)].
9 K.A. Matveev, L.I. Glazman, Phys. Rev. B 54, 10339 (1996).

10 C.W.J. Beenakker, Phys. Rev. B 44, 1646 (1991).

11 M.L. Mehta, Random Matrices, 2nd Ed. (Academic Press, London, 1991).

12 R. Denton, B. Mühlschlegel, D.J. Scalapino, Phys. Rev. B 7, 3589 (1973).

13 F.D.M. Haldane, J. Phys. C: Solid State Phys. 14, 2585 (1981).

14 A.H. MacDonald, in Quantum Transport in Semiconductor Submicron Structures, edited by B. Kramer, NATO ASI, Ser. E, Vol. 326 (Kluwer, Dordrecht, 1996), pp. 63-91.

15 See, e.g., L.I. Glazman, in Quantum Mesoscopic Phenomena and Mesoscopic Devices in Microelectronics, edited by Igor O. Kulik and Recai Ellialtioğlu, NATO ASI, Ser. C, Vol. 559 (Kluwer, Dordrecht, 2000), pp. 105-128.

16 I.L. Aleiner, P.W. Brouwer, L.I. Glazman, Phys. Rep. 358, 309 (2002). 